\title{
Transvaginal Color Doppler in the Assessment of Cervical Carcinoma and Pre-Cancer: Evidence from a Case Control Study Using Colour Doppler Ultrasonography Pulsatility Index of Uterine Vasculature
}

\author{
S. H. Dodampahala"1, S. N. Jayakody ${ }^{2}$, W. C. C. Gunathilake ${ }^{3}$, A. N. Rahubaddha1, \\ S. K. Dodampahala ${ }^{4}$ \\ ${ }^{1}$ Department of Obstetrics and Gynecology, Faculty of Medicine, University of Colombo, Colombo, Sri Lanka \\ ${ }^{2}$ Post Graduate Institute of Medicine, Colombo, Sri Lanka \\ ${ }^{3}$ De Soysa Hospital for Women, Colombo, Sri Lanka \\ ${ }^{4}$ Australian College of Business Technology in Sri Lanka, Colombo, Sri Lanka \\ Email: hemdodam@gmail.com
}

Received 14 July 2016; accepted 12 August 2016; published 16 August 2016

Copyright (C) 2016 by authors and Scientific Research Publishing Inc.

This work is licensed under the Creative Commons Attribution International License (CC BY).

http://creativecommons.org/licenses/by/4.0/

c) (†) Open Access

\begin{abstract}
Introduction: Transvaginal colour Doppler is a non-invasive ultrasound-based technique that allows an in-vivo assessment of tumor vascularization. Several researches done in the last decade have evaluated the role of this technique in assessing carcinoma of the cervix. However there is a significant paucity of literature linked to pre-malignant lesions of the cervix and its diagnosis based on transvaginal colour doppler and its pulstality index (PI). In this paper we present a case control study conducted to assess the validity of transvaginal colour doppler scan and PI in diagnosing malignant and pre-malignant lesions of the cervix. Methodology: A case control study with a total sample of 57 women was conducted at the Nawaloka Hospital for duration of two years. 19 cases of recently diagnosed patients with cervical carcinoma, using cytobrush and colposcopy guided biopsy, evaluated by a consultant histopathologist, were recruited. Staging was done according to FIGO classification. 40 age-matched patients with histologically confirmed normal cervix were selected as controls and 2 were excluded from the study due to the presence of cervical infection. All cases and controls were subjected to transvaginal colour doppler sonography using a


$5 \mathrm{MHz}$ 3D probe using GEvolusion6 US machine. The machine was also set for high pass filter at $100 \mathrm{~Hz}$ to eliminate low frequency signals occurring from vessel-wall motion. Cervical colour doppler ultrasonography pattern was captured in each case of malignancy, pre-malignancy and controls, and pulstality index (PI) was assessed by the lead gynecologist accredited with ISUOG standards. The highest PI was assessed and recorded in the descending branch of the uterine artery. Results: Women's mean age was 55.5 years (range 35 - 78). According to clinical staging, there were 4 females with carcinoma in situ, 4 Females with Stage I a, 3 with Stage I b, 4 with Stage II a and 4 with Stage III a. Histologic examination revealed 13 women with squamous cell carcinoma, 2 women with adenocarcinoma and 4 with carcinoma in situ (CIN III) of the cervix. Mean PI value for the cases were 1.94 (range 1.2 - 3.1) and the mean PI value for the controls were 0.805 (range 0.3 - 1.5). Neovascularization was markedly seen in the patients with invasive cervical carcinoma with a PI ranging between 1.99 and 3.10. Chi square test results showed a very high statistically significant difference of PI values between cases and controls ( $p$ value $<0.0001)$. An ROC analysis revealed the optimal cut-off value of PI at 1.475 with a sensitivity of $89.5 \%$ and a specificity of $\mathbf{9 3 . 5 \%}$. Conclusion: Transvaginal doppler ultrasonography and PI of the descending cervical branch of the uterine artery and its branch vasculature allow a non-invasive assessment of tumor vascularization in cervical carcinoma and pre-malignancy. PI with a cut off value of 1.475 , can be reliably used to detect and screen pre-malignancies and malignancies at a relatively early stage with a sensitivity of $89.5 \%$ and a specificity of $93.5 \%$ enabling further definitive evaluation, in a routine gynecology trans vaginal ultrasound assessment.

\section{Keywords}

Carcinoma of the Cervix, Pre-Malignancy of the Cervix, Transvaginal Cervical Colourdoppler, Pulstality Index

\section{Introduction}

Carcinoma of the cervix is the second most common female-specific cancer worldwide [1]. 80\% of all cervical cancers occur in women in the developing countries leading to significant morbidity and mortality [1]. The main prognostic factor being the stage of cancer at which it is diagnosed, with treatment, the five-year relative survival rate for the earliest stage of invasive cervical cancer is $92 \%$. It reduces to $17 \%$ for stage IV disease [2]. Hence it is of paramount importance to screen and detect cervical cancer in its early stages.

Angiogenesis is the production of new vessels in a specific area. Specifically, in cancer of the cervix, angiogenesis has been shown to be an independent prognostic factor [3] and to predict recurrence [4]. Transvaginal Doppler ultrasound allows an in-vivo non-invasive assessment of tumor angiogenesis [5].

With the advance of diagnostic ultrasonography, clinical use of ultrasonic technology has increased as a way to visualize changes in the uterine vasculature, in the assessment of the development of the basal layer of the endometrium. Although there are numbers of studies assessing the endometrial blood flow versus endometrial receptivity and fertility there is a paucity of such assessment in detecting cervical malignancies which remain an unresolved cause of mortality among sexually active women [6]. Ultrasonographic findings indicating changes in the cervical blood flow in relation to cervical malignancy have not yet been widely acknowledged. Therefore, in this case control study, we aim to examine the value of ultrasongraphic technology in the assessment of cervical blood flow and clarify its relationship with the occurrence of pre-malignancy and malignancy compared to women with normal cervical cytology.

Doppler ultrasound may be used in several modes: Pulsed Doppler, Colourdoppler and Power Doppler. In general a Doppler scan allows the assessment of blood flow velocity and resistance in a given vessel at a given moment. Color Doppler uses the same principle and allows the visualization of vessels by color-coding. This method is useful to determine the presence of vessels and provides information about the amount of vessels, their distribution and arrangement [7].

Pulsatality index is a measure of the variability of blood velocity in a vessel, equal to the difference between 
the peak systolic and minimum diastolic velocities divided by the mean velocity during the cardiac cycle [8]. It was noted during a through literature survey that there is a significant paucity of studies carried out to determine the use of transvaginal doppler ultrasonography to detect pre-malignant lesions of the cervix. Therefore it is timely that a study in this aspect was conducted specially in a developing country such as Sri Lanka where there is a significant burden on national healthcare services due to cervical cancer, compared to the developed countries.

It is observed in routine gynecology practice that some patients may not reveal correct information about their cervical screening. It is also observed that certain women show marked reluctance to undergo invasive cytological assessment, colposcopy and guided biopsy. However most of the women grant consent for non-invasive transabdominal or transvaginal assessment of the uterus, cervix and ovaries. This method of noninvasive assessment of the cervix for malignancies and pre-malignancies using colour Doppler ultrasonography, would be a reliable tool in detecting such lesions in future gynecology.

\section{Methodology}

A case control study was conducted to assess the validity of Doppler ultrasound technique in the diagnosis of cervical carcinoma.

Study setting-The study was conducted at Nawaloka Hospitals PLC, which is a privately owned tertiary care referral center for obstetrics and gynecology patients.

Study period: The study was conducted for a period of two years from $1^{\text {st }}$ January 2013 to $31^{\text {st }}$ December 2015.

Study population: All menstruating cases and controls were scanned around day 6 to day 10 of their respective menstrual cycle. Cases were recently diagnosed patients with cervical carcinoma using cytobrush and colposcopy guided biopsy evaluated by a consultant histopathologist. Staging was done according to FIGO classification. 19 cases including 2 patients with intact hymen were selected adhering to the above case definition. 40 age-matched patients with histologically confirmed normal cervix were selected as controls. Two were excluded from the study due to the presence of cervical infection. The purpose of the study was explained to the study subjects and informed written consent was obtained.

All cases and controls were examined using transvaginal colour doppler sonography using $5 \mathrm{MHz}$ 3D probe using GEvolusion 6 US machine (US patent). The machine was also set for high pass filter at $100 \mathrm{~Hz}$ to eliminate low frequency signals occurring from vessel-wall motion. The cervical colour doppler ultrasonography pattern was captured in each case of malignancy, pre-malignancy and normal cases (Figure 1(a), Figure 1(b)).

The colour Doppler images were captured and pulstality index (PI) was assessed by the lead gynecologist accredited with ISUOG standards.

Data analysis: Statistical Package for Social Sciences (SPSS) version 17 for Microsoft Windows was used for statistical analysis. Cases and controls were categorized according to PI values. Significance of the PI value among the cases and controls were then assessed using Chi square test. P value of $<0.05$ was taken as a significant difference.

Cut-off value was derived using Receiver Operating characteristics (ROC) curve analysis for categorizing patients on having or not having cervical cancer based on PI value.

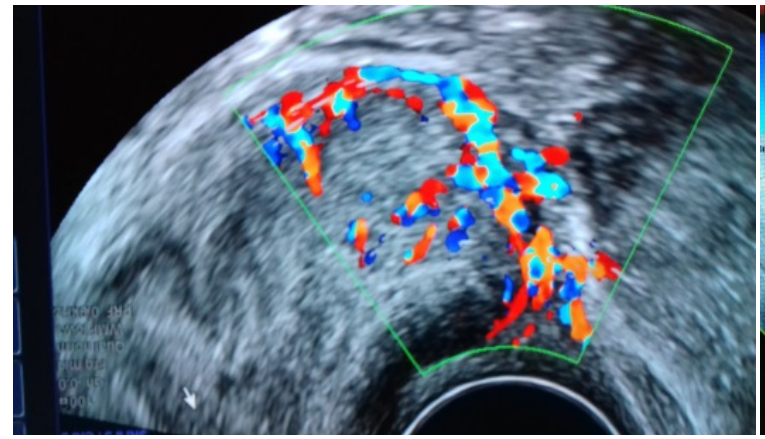

(a)

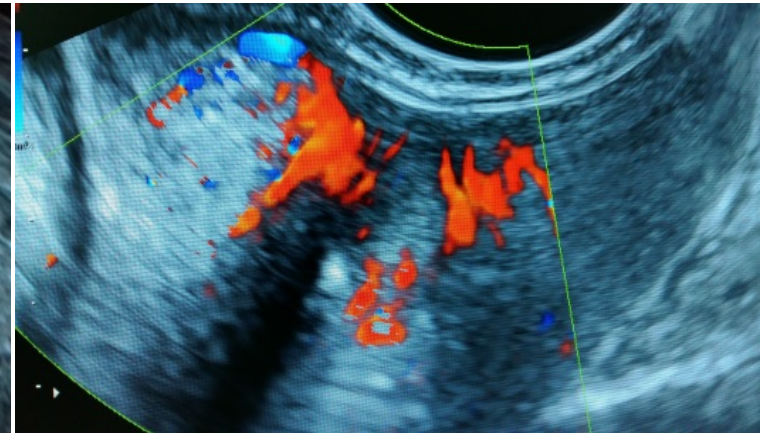

(b)

Figure 1. (a) Neovascularization pattern of invasive cervical cancer; (b) Neovascularization pattern of CIN III. 


\section{Results}

The mean age was 55.5 years (range 35 - 78). According to clinical staging, there were 4 females in Stage I a, 3 females in Stage I b, 4 females in Stage II a, 4 females in Stage III a. Four women had carcinoma in situ. Histological examination revealed 13 women with squamous cell carcinoma, 2 women with adenocarcinoma and 4 with carcinoma in situ (CIN III) of the cervix (Table 1).

Mean PI value for the cases was 1.94 (range 1.2 - 3.1) and the mean PI value for the controls was 0.805 (range 0.3 - 1.5). Neovascularization was markedly seen in the patients with invasive cervical carcinoma with PI ranging between 1.99 and 3.10 (Figure 2).

All patients with FIGO stage I to II were offered Wertheim's Hysterectomy with lymph node dissection, removal of the cuff of vagina and subsequent brachytherapy. Among the patients with FIGO stage III lesions, one defaulted to undergo ayurvedic treatment. Other three patients were referred to specialized cancer hospital for radical pelvic exenteration and radiotherapy. Among the four patients with CIN III, one underwent hysterectomy and three others had knife cone excision and follow up.

Chi square test results showed a very high statistically significant difference of PI values among cases and controls ( $\mathrm{p}$ value $<0.0001$ ).

\section{Histogram}

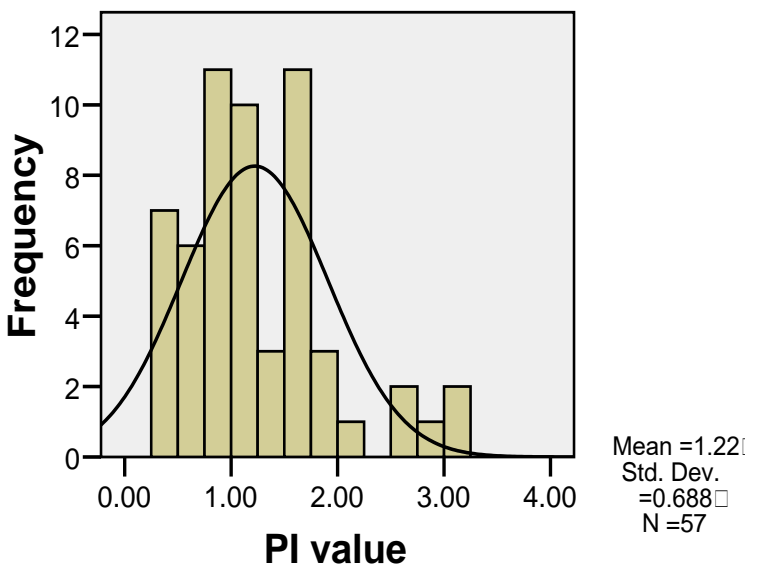

Figure 2. Frequency distribution of PI values of the total sample.

Table 1. General characteristics of the study group.

\begin{tabular}{lc}
\hline \multicolumn{1}{c}{ Characteristic } & Frequency (n)/\% \\
\hline Total & $57.5(35-78)$ \\
Mean age (yrs)/range & \\
FIGO stage & $4(21 \%)$ \\
Carcinoma in situ & $4(21 \%)$ \\
I a & $3(15.7 \%)$ \\
I b & $4(21 \%)$ \\
II a & $4(21 \%)$ \\
III a & \\
Histology & $13(68.7 \%)$ \\
Squamous cell & $2(10.5 \%)$ \\
Adenocarcinoma & $4(21 \%)$ \\
CIN III & \\
\hline
\end{tabular}


In the ROC analysis, PI value obtained by each individual was taken as the test variable and the presence or absence of cervical carcinoma confirmed by histology as the gold standard. Using these values, an ROC curve was plotted against sensitivity and false positive rates (1-specificity) of the PI values in the sample (Figure 3). Optimal cut off value was obtained by the maximum length from the area under the curve to the diagonal line.

The area under the curve which is a global measure of test performance was 0.946 . The optimal cut-off value of the test was 1.475 with a sensitivity of $89.5 \%$ and a specificity of $93.5 \%$.

\section{Discussion}

The interest in using transvaginal colour doppler ultrasonography to detect cervical cancer has been increasing during the last two decades. The pros and cons of different modes of Doppler ultrasound scans are well documented in the literature [9]. However in our personal experience, the use of Doppler ultrasonography to confirm a suspected cervical lesion is less common, especially in a developing country such as Sri Lanka. In some studies it has been suggested that colour signals may be identified in $100 \%$ of cervical cancers [8]. Studies have also suggested that blood flow, as assessed by transvaginal color Doppler, correlated with some tumor characteristics that could lead to prognostication purposes and determination of response to treatment [10]. However these characteristics are still in doubt and require further studies to arrive at a consensus. These studies are most likely to bring ease to the gynecologists and oncologists when making decisions with regard to treatment protocols. However there is a significant paucity of literature linked to pre-malignant lesions of the cervix and its diagnosis based on transvaginal colour Doppler and its pulstality index (PI).

Although we did not have a large sample of patients these results are encouraging and reassuring. According to the findings colour Doppler ultrasonography and PI of uterine vessels would give a good prediction of neovascularization of cervical malignancy and pre-malignancy. It was also shown in the study that the patients with normal smear cytology have significantly less vasculature.

In our study two controls were excluded from the study on the basis that any infection or inflammation mediated vascular response may result in creating a false positive to the study [9].

In this particular study we used colour Doppler ultrasonography to the highest effect to detect high blood flow and neo-vasculrization in a cervical cancer. This ultimately paved the path to the diagnosis of cervical cancerresulting in curative resections where possible.

This assessment was uniquely beneficial to two unmarried females with intact hymen. They both suffered stage II adenocarcinoma of the cervix and had not undergone a cervical smear as they had intact hymen. A finding of high pulstality index in these symptomatic women allowed us to investigate further with colposcopy and cytobrush after counseling them. These patients' descending branch of the uterine artery Dopplers were assessed by abdominal ultrasound scan (Figure 4). It is our understanding during this study that Doppler assessment of the uterine vessels would give immense information about a possible malignancy or pre-malignancy in the cervix in an unmarried woman with an intact hymen especially seen in Southeast Asian culture.

\section{ROC Curve}

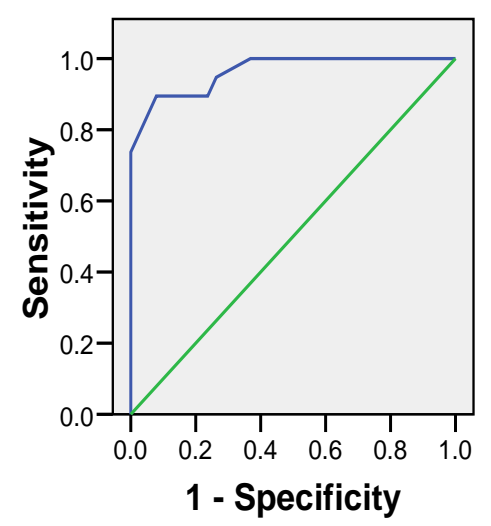

Diagonal segments are produced by ties.

Figure 3. ROC curve. 


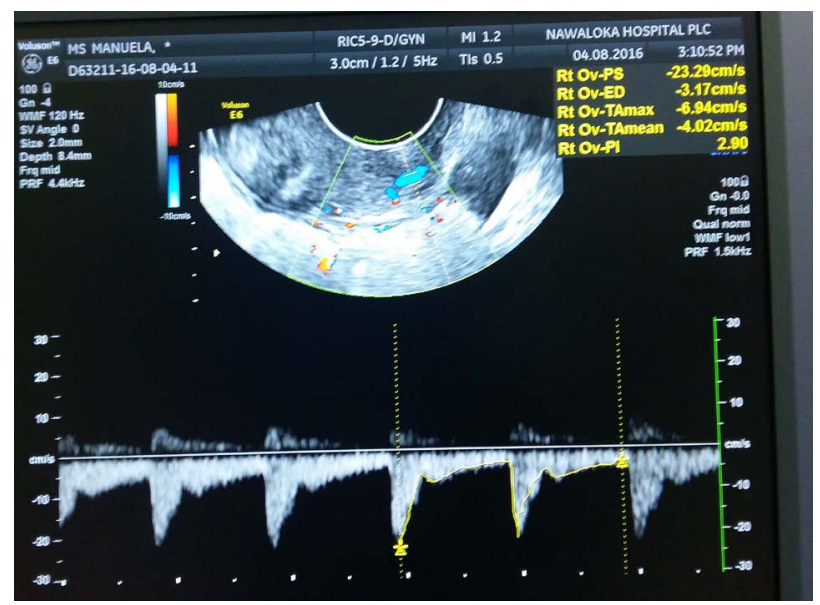

Figure 4. Abdominal uterine vasculature with high PI (pulstality index).

\section{Conclusion}

Transvaginal colour Doppler ultrasonography allows a non-invasive assessment of tumor vascularization in cervical carcinoma. It can be used to detect and diagnose cancers at a relatively early stage enabling curative resection and thus offering a higher survival rate. With the advancement of studies, transvaginal colour Doppler ultrasonography may also be used for prognostication and to decide upon treatment options. In this study, we postulated that this non-invasive technique can be useful as an adjunct in the management of women with abnormal pap smear. Further abnormal findings of colour Doppler of uterine vessels would be an indication for colposcopic assessment and biopsy. This study clearly illustrates the unique benefits of using abdominal uterine artery colour Doppler assessment in women with intact hymen and in women who decline vaginal examination and cervical smear screening.

\section{Declaration of Conflicting Interest}

The authors declare that there are no conflicts of interest.

\section{Funding}

This research received no specific grant from any funding agency in the public, commercial, or not for-profit sectors.

\section{References}

[1] International Agency for Research on Cancer (2014) World Cancer Report 2014 (International Agency for Research on Cancer). World Health Organization, Geneva.

[2] Sala, E., Wakely, S., Senior, E., et al. (2007) MRI of Malignant Neoplasms of the Uterine Corpus and Cervix. American Journal of Roentgenology, 188, 1577-1587. http://dx.doi.org/10.2214/AJR.06.1196

[3] Obermair, A., Wanner, C., Bilgi, S., Speiser, P., Kaider, A., Reinthaller, A., Leodolter, S. and Gitsch, G. (1998) Tumor Angiogenesis in Stage IB Cervical Cancer: Correlation of Microvessel Density with Survival. American Journal of Obstetrics \& Gynecology, 178, 314-319. http://dx.doi.org/10.1016/S0002-9378(98)80018-5

[4] Dinh, T.V., Hannigan, E.V. and Smith, E.R. (1996) Tumor Angiogenesis as Predictor of Recurrence in Stage Ib Squamous Cell Carcinoma of the Cervix. Gynecologic Oncology, 87, 751-754. http://dx.doi.org/10.1016/0029-7844(96)00039-7

[5] Cosgrove, D. (2003) Angiogenesis Imaging-Ultrasound. The British Journal of Radiology, 76, S43-S49. http://dx.doi.org/10.1259/bjr/86364648

[6] Wang, L.N., Qiao, J., Li, R., Zhen, X.M. and Liu, Z.H. (2010) Role of Endometrial Blood Flow Assessment with Color Doppler Energy in Predicting Pregnancy Outcome of IVF-ET Cycles. Reproductive Biology and Endocrinology, 8, 122. http://dx.doi.org/10.1186/1477-7827-8-122

[7] Alcázar, J.L. and Jurado, M. (1999) Transvaginal Color Doppler for Predicting Pathological Response to Preoperative 
Chemoradiation in Locally Advanced Cervical Carcinoma: A Preliminary Study. Ultrasound in Medicine and Biology, 25, 1041-1045. http://dx.doi.org/10.1016/S0301-5629(99)00063-0

[8] Sciscione, A.C. and Hayes, E.J. (2009) Uterine Artery Doppler Flow Studies in Obstetric Practice. American Journal of Obstetrics and Gynecology, 201, 121-126. http://dx.doi.org/10.1016/j.ajog.2009.03.027

[9] Alcazar, J.L. (2005) Transvaginal Color Doppler in the Assessment of Cervical Carcinoma. Cancer Therapy, 3, 139146.

[10] Hsu, K.F., Su, J.M., Huang, S.C., Cheng, Y.M., Kang, C.Y., Shen, M.R., Chang, F.M. and Chou, C.Y. (2004) ThreeDimensional Power Doppler Imaging of Early-Stage Cervical Cancer. Ultrasound in Obstetrics \& Gynecology, 24, 664-671. http://dx.doi.org/10.1002/uog.1756

\section{Submit or recommend next manuscript to SCIRP and we will provide best service for you:}

Accepting pre-submission inquiries through Email, Facebook, LinkedIn, Twitter, etc.

A wide selection of journals (inclusive of 9 subjects, more than 200 journals)

Providing 24-hour high-quality service

User-friendly online submission system

Fair and swift peer-review system

Efficient typesetting and proofreading procedure

Display of the result of downloads and visits, as well as the number of cited articles

Maximum dissemination of your research work

Submit your manuscript at: http://papersubmission.scirp.org/ 